\title{
Three-loop evolution equation for (non-singlet) leading-twist operators
}

\author{
Matthias Strohmaier* $\dagger$ \\ Institut für Theoretische Physik, Universität Regensburg, D-93040 Regensburg, Germany \\ E-mail: matthias.strohmaier@ur.de
}

We present the three-loop evolution kernel entering the evolution equation of non-singlet leading twist operators. We give an approximate expression for the evolution kernel of non-local operators. We show how to transform the evolution equation from the light-cone representation into the language of local operators. We present the exact anomalous dimension matrix at NNLO for a certain set of local operators, motivated by the conformal expansion of distribution amplitudes.

QCD Evolution 2017

22-26 May, 2017

Jefferson Lab, Newport News, VA - USA

\footnotetext{
* Speaker.

${ }^{\dagger}$ The author thanks V. M. Braun, A. N. Manashov and S. Moch for the collaboration on this project.
} 


\section{Introduction}

During the last decade there have been made great achievements in experimental particle physics, not only concerning the accuracy but also concerning the variety of accessible processes. Within the near future even more improvements can be expected as due to the current JLAB upgrade [1] or the planned EIC [2]. On the theoretical side this offers on the one hand great opportunities, however, on the other hand there arises of course a strong demand for a higher precision in theoretical description. A Next-to-next-to-leading order (NNLO) analysis is becoming standard accuracy in most fields [3].

The theoretical description is usually performed using the factorization theorem, i.e. the separation of the process into a perturbatively accessible (hard) part and a non-perturbative (soft) part. The soft part is typically given in terms of so-called generalized parton distributions (GPDs) or distribution amplitudes (DAs). The scale dependence of these GPDs and DAs is governed by evolution equations. For processes with incoming and outgoing particles carrying the same momenta the NNLO-prescription is available for quite some times [4], recently partial NNNLO results have been published [5]. For processes with non-zero momentum transfer the calculation is much more challenging as mixing between operators with different number of derivatives need to be taken into account. Despite impressive progresses in the field of higher-order calculations there are still only NLO results available [6-8].

It is well known that conformal symmetry allows one to fix the off-forward dynamics in a simplified way [9]. To do so one considers QCD in non-integer $d=4-2 \varepsilon$ dimensions at a critical fix-point $\beta\left(a_{*}\right)=0$. For this theory one can expect that Poincare-symmetry gets enhanced by scale- and conformal-symmetry. Therefore, in the collinear limit, there must exist three symmetry generators which commute with the evolution equations [10]. These generators, however, are not the canonical ones but get non-trivial perturbative corrections. The benefit by this method is, that the knowledge of $(\ell-1)$-loop corrections to the symmetry generators is sufficient to fix the evolution kernel at $(\ell)$-loops. In MS-like renormalization schemes the inhomogeneous part of the evolution equation - the evolution kernel - is not sensitive to the space-time dimension. Thus we can conclude that the evolution kernel we find in this way in the modified conformal theory at the critical point must be the same as in the physical $d=4$ QCD.

Using this method and the recent two-loop results for the conformal symmetry generators in the modified theory in $d=4-2 \varepsilon$ dimension [11] we derive explicit results for the three-loop evolution kernel in the $\overline{\mathrm{MS}}$-scheme.

\section{Evolution equations for leading-twist operators}

Let us consider the renormalized twist-2 light-ray operator

$$
[\mathscr{O}]\left(x ; z_{1}, z_{2}\right)=Z \bar{q}\left(x+z_{1} n\right) h q\left(x+z_{2} n\right),
$$

where a gauge link is tacitly assumed between the quark fields (of different flavors) with a light-like separation $n^{2}=0$. This operator can be viewed as a generating function for renormalized twist-2 
local operators

$$
[\mathscr{O}]\left(x ; z_{1}, z_{2}\right) \equiv \sum_{m, k} \tilde{P}_{m k}\left(z_{1}, z_{2}\right)\left[O_{m k}\right](x), \quad\left[O_{m k}\right]=\bar{q}(x) P_{m k}(\overleftarrow{D}, \vec{D}) q(x)
$$

where $D_{\mu}=\partial_{\mu}-i g A_{\mu}$ is the covariant derivative and $P_{m k}(x, y)$ and $\tilde{P}_{m k}(x, y)$ are polynomials of degree $m+k$, which satisfy the orthogonality relation

$$
\left.P_{m k}\left(\partial_{x}, \partial_{y}\right) \tilde{P}_{m^{\prime} k^{\prime}}(x, y)\right|_{x=y=0}=\delta_{m m^{\prime}} \delta_{k k^{\prime}}
$$

The relation (2.3) can be seen as a definition of a scalar product for these polynomials.

The square brackets denote renormalization in $\overline{\mathrm{MS}}$-scheme

$$
[\mathscr{O}]\left(x ; z_{1}, z_{2}\right)=\mathbb{Z} \mathscr{O}\left(x ; z_{1}, z_{2}\right), \quad\left[O_{m k}\right](x)=\sum_{m^{\prime} k^{\prime}} Z_{m k}^{m^{\prime} k^{\prime}} O_{m^{\prime} k^{\prime}}(x),
$$

where the operator $\mathbb{Z}$ is an integral operator acting on the light-cone coordinates $\left(z_{1}, z_{2}\right)$ and $Z_{m k}^{m^{\prime} k^{\prime}}$ is a matrix describing the mixing of local operators under renormalization. Both are given by a Laurent series in $\varepsilon$

$$
Z=1+\sum_{k=0} \frac{Z_{k}(a)}{\varepsilon^{k}}, \quad Z_{k}(a)=\sum_{\ell=k}^{\infty} a^{\ell} Z_{k}^{(\ell)}
$$

Throughout the work we denote the strong coupling by $a=\frac{g^{2}}{(4 \pi)^{2}}$.

The evolution equations for the twist- 2 operators take the form

$$
\left(\mu \frac{\mathrm{d}}{\mathrm{d} \mu}+\mathbb{H}\right)[\mathscr{O}]\left(x ; z_{1}, z_{2}\right)=0, \quad \sum_{m^{\prime} k^{\prime}}\left(\mu \frac{\mathrm{d}}{\mathrm{d} \mu} \delta_{m m^{\prime}}^{k k^{\prime}}+\gamma_{m k}^{m^{\prime} k^{\prime}}\right)\left[O_{m^{\prime} k^{\prime}}\right]\left(x ; z_{1}, z_{2}\right)=0,
$$

where $\mu$ is the renormalization scale, the evolution kernel $\mathbb{H}(a)$ is an integral operator and $\gamma_{m m^{\prime}}^{k k^{\prime}}$ is called anomalous dimension matrix. They are related to the renormalization factors by the logarithmic derivative $-\frac{\mathrm{d}}{\mathrm{d} \ln \mu} \ln Z$, i.e.

$$
\mathbb{H}=2 \sum_{\ell} \ell a^{\ell} \mathbb{Z}_{1}^{(\ell)}, \quad \quad \gamma_{m k}^{m^{\prime} k^{\prime}}=2 \sum_{\ell} \ell a^{\ell} Z_{1}^{(\ell)}{ }^{m^{\prime} k^{\prime}} .
$$

By the means of the scalar product (2.3) one easily finds a connection between the evolution kernel and the anomalous dimension matrix

$$
\gamma_{m k}^{m^{\prime} k^{\prime}}=\left.P_{m k}\left(\partial_{1}, \partial_{2}\right) \mathbb{H} \tilde{P}_{m^{\prime} k^{\prime}}\left(z_{1}, z_{2}\right)\right|_{z_{1}=z_{2}=0}
$$

The standard derivation for the inhomogeneous part of the evolution equation as residue of the renormalization constants is notoriously difficult, as Eq. (2.7) relates both quantities at the same accuracy in the perturbative expansion. As already mentioned conformal symmetry of a modified theory allows for an alternative and simpler derivation of the evolution equations, see e.g. $[6,7,9,12]$ for details. In the following we will use this technique to derive both anomalous dimensions and evolution kernel to NNLO accuracy. 


\section{Method and results: light-cone formulation}

Let us consider QCD in $d=4-2 \varepsilon$ dimensions. This theory is known to posses a non-trivial critical point for a large number of fermion flavors $[13,14]$. This critical point $a_{*}$ is defined as the root of the $\beta$-function

$$
\beta\left(a_{*}\right)=-2 a_{*}\left(\varepsilon+\bar{\beta}\left(a_{*}\right)\right)=0, \quad \bar{\beta}(a)=a \beta_{0}+a^{2} \beta_{1}+O\left(a^{3}\right),
$$

and reads

$$
a_{*}=-\frac{\varepsilon}{\beta_{0}}-\left(\frac{\varepsilon}{\beta_{0}}\right)^{2} \frac{\beta_{1}}{\beta_{0}}+O\left(\varepsilon^{3}\right),
$$

where

$$
\beta_{0}=11-\frac{2}{3} n_{f}, \quad \beta_{1}=102-\frac{38}{3} n_{f} .
$$

Fixing the coupling to its critical value we define a theory that is invariant under conformal symmetry transformations. ${ }^{1}$

In this modified theory the three collinear conformal symmetry generators must satisfy the SL(2)-algebra

$$
\left[S_{0}, S_{ \pm}\right]= \pm S_{ \pm}, \quad\left[S_{+}, S_{-}\right]=2 S_{0} .
$$

However, compared to the canonical symmetry generators the exact symmetry generators need to be modified by quantum corrections

$$
S_{ \pm, 0}=S_{ \pm, 0}^{(0)}+\Delta S_{ \pm, 0}\left(a_{*}\right)
$$

which can be calculated order by order in perturbation theory employing conformal ward identities. Details on the method and the corresponding results to one- and two-loop accuracy can be found in $[11,12]$.

The constraint (3.4) turns out to be equivalent to the statement, that the three generators commute with the evolution kernel

$$
\left[S_{ \pm, 0}\left(a_{*}\right), \mathbb{H}\left(a_{*}\right)\right]=0
$$

For two of them, $S_{-}$and $S_{0}$, this statement is trivial, for the generator of special conformal transformations $S_{+}$the equation

$$
\left[S_{+}^{(0)}, \mathbb{H}\left(a_{*}\right)\right]=\left[\mathbb{H}\left(a_{*}\right), \Delta S_{+}\left(a_{*}\right)\right]
$$

however contains non-trivial information. A perturbative expansion

$$
\mathbb{H}\left(a_{*}\right)=\sum_{\ell} a_{*}^{\ell} \mathbb{H}^{(\ell)}, \quad \Delta S_{+}\left(a_{*}\right)=\sum_{\ell} a_{*}^{\ell} \Delta S_{+}^{(\ell)},
$$

\footnotetext{
${ }^{1}$ In gauge theories, only correlators of gauge-invariant operators will transform properly under conformal transformations
} 
reveals that the commutator of $S_{+}^{(0)}$ and the $\ell$-loop evolution kernel is given by products of operators of order $k \leq \ell-1$ :

$$
\left[S_{+}^{(0)}, \mathbb{H}^{(\ell)}\right]=\sum_{k=1}^{\ell-1}\left[\mathbb{H}^{(k)}, \Delta S_{+}^{(\ell-k)}\right] .
$$

This equation fixes the $\ell$-loop evolution kernel up to solutions of the homogeneous equation

$$
\left[S_{+}^{(0)}, \mathbb{H}_{\text {inv }}^{(\ell)}\right]=0
$$

which we will call (canonically) invariant part. For the generic form of the evolution kernel

$$
\mathbb{H} \mathscr{O}\left(z_{1}, z_{2}\right)=\int_{0}^{1} d \alpha \int_{0}^{\bar{\alpha}} d \beta h(\alpha, \beta) \mathscr{O}\left(z_{12}^{\alpha}, z_{21}^{\beta}\right), \quad z_{12}^{\alpha}=\bar{\alpha} z_{1}+\alpha z_{2},
$$

with $\bar{\alpha}=1-\alpha$, the 1.h.s. of Eqs. (3.9) and (3.10) can be written as a first order partial differential equation for the kernel function $h(\alpha, \beta)$. The homogeneous equation is solved by any function of the conformal ratio $\tau=\frac{\alpha \beta}{\bar{\alpha} \bar{\beta}}$, thus we split

$$
\mathbb{H}=\mathbb{H}_{\mathrm{inv}}+\mathbb{H}_{\mathrm{ninv}}, \quad h(\alpha, \beta)=h_{\mathrm{inv}}(\tau)+h_{\text {ninv }}(\alpha, \beta) .
$$

Consequently, the non-invariant part $\mathbb{H}_{\text {ninv }}$ is found as solution of the inhomogeneous equation (3.9) and the invariant part, which has only one degree of freedom, needs to be chosen such that the evolution kernel reproduces the spectrum of forward anomalous dimensions

$$
\mathbb{H}\left(z_{1}-z_{2}\right)^{N}=\gamma_{N}\left(z_{1}-z_{2}\right)^{N}
$$

which is known to four-loop accuracy [5].

\subsection{Non-invariant part}

It was shown that the correction to the generator of special (collinear) conformal transformations takes the form

$$
\Delta S_{+}=\left(z_{1}+z_{2}\right)(\bar{\beta}(a)+\mathbb{H}(a))+\left(z_{1}-z_{2}\right) \Delta(a)
$$

where $\bar{\beta}(a)=-\frac{\beta(a)}{2 a}$ and the operator $\Delta(a)$ is called conformal anomaly, is specific for gauge theories and contains all non-trivial corrections.

The determination of the non-invariant part is done in a two-step process: First we find a transformation

$$
\mathbb{U}:\left[\mathscr{O}\left(z_{1}, z_{2}\right)\right] \mapsto\left[\mathscr{O}\left(z_{1}, z_{2}\right)\right]^{\mathbb{U}}=\mathbb{U}\left[\mathscr{O}\left(z_{1}, z_{2}\right)\right],
$$

such that the action of the operator $S_{+}(a)$ takes the form

$$
\mathbf{S}_{+}(a)=\mathbb{U}^{-1} S_{+}(a) \mathbb{U}=S_{+}^{(0)}+\left(z_{1}+z_{2}\right)\left(\beta(a)+\frac{1}{2} \mathbb{U}^{-1} \mathbb{H}(a) \mathbb{U}\right) .
$$

Note that in $\mathbf{S}_{+}(a)$ the anomaly $\Delta(a)$ is absent and it has "almost canonical" form. We parametrize

$$
\mathbb{U}(a)=e^{\mathbb{X}(a)}=e^{a \mathbb{X}^{(1)}+a^{2} \mathbb{X}^{(2)}+O\left(a^{3}\right)},
$$


and compare Eq. (3.16) with (3.14) to receive the following constraints for the operator $\mathbb{X}$

$$
\begin{aligned}
& {\left[S_{+}^{(0)}, \mathbb{X}^{(1)}\right]=z_{12} \Delta^{(1)}} \\
& {\left[S_{+}^{(0)}, \mathbb{X}^{(2)}\right]=z_{12} \Delta^{(2)}+\left[\mathbb{X}^{(1)}, z_{1}+z_{2}\right]\left(\beta_{0}+\frac{1}{2} \mathbb{H}^{(1)}\right)+\frac{1}{2}\left[\mathbb{X}^{(1)}, z_{12} \Delta^{(1)}\right] .}
\end{aligned}
$$

These equations fix the operator $\mathbb{X}(a)$ only up to canonically invariant pieces. The transformation (3.15) represents a finite renormalization scheme transformation, i.e. the operators $[\mathscr{O}]^{\mathbb{U}}$ satisfy the following evolution equation

$$
\left(M \partial_{M}+\beta(a) \partial_{a}+\mathbb{U}^{-1} \mathbb{H}(a) \mathbb{U}-\beta(a)(\partial \mathbb{U}) \mathbb{U}^{-1)}\right)\left[\mathscr{O}\left(z_{1}, z_{2}\right)\right]^{\mathbb{U}}=0 .
$$

One easily notices that the evolution kernel in this scheme

$$
\mathbf{H}(a)=\mathbb{U}^{-1} \mathbb{H}(a) \mathbb{U},
$$

has the same spectrum as in $\overline{\mathrm{MS}}$-scheme. It must satisfy the constraint

$$
\left[\mathbf{S}_{\alpha}(a), \mathbf{H}(a)\right]=0
$$

which turns out to have a much simpler structure and solution than the Eq. (3.9) in $\overline{\mathrm{MS}}$-scheme . Order by order in a perturbative expansion we receive the following set of equations

$$
\begin{aligned}
& {\left[S_{+}^{(0)}, \mathbf{H}^{(1)}\right]=0,} \\
& {\left[S_{+}^{(0)}, \mathbf{H}^{(2)}\right]=\left[\mathbf{H}^{(1)}, z_{1}+z_{2}\right]\left(\beta_{0}+\frac{1}{2} \mathbf{H}^{(1)}\right),} \\
& {\left[S_{+}^{(0)}, \mathbf{H}^{(3)}\right]=\left[\mathbf{H}^{(1)}, z_{1}+z_{2}\right]\left(\beta_{1}+\frac{1}{2} \mathbf{H}^{(2)}\right)+\left[\mathbf{H}^{(2)}, z_{1}+z_{2}\right]\left(\beta_{0}+\frac{1}{2} \mathbf{H}^{(1)}\right) .}
\end{aligned}
$$

These equations are solved by

$$
\begin{aligned}
& \mathbf{H}^{(1)}=\mathbf{H}_{\mathrm{inv}}^{(1)}, \\
& \mathbf{H}^{(2)}=\mathbf{H}_{\mathrm{inv}}^{(2)}+\mathbb{T}^{(1)}\left(\beta_{0}+\frac{1}{2} \mathbf{H}_{\mathrm{inv}}^{(1)}\right), \\
& \mathbf{H}^{(3)}=\mathbf{H}_{\mathrm{inv}}^{(3)}+\mathbb{T}^{(1)}\left(\beta_{1}+\frac{1}{2} \mathbf{H}_{\mathrm{inv}}^{(2)}\right)+\mathbb{T}_{1}^{(1)}\left(\beta_{0}+\frac{1}{2} \mathbf{H}_{\mathrm{inv}}^{(1)}\right)^{2}+\left(\mathbb{T}^{(2)}+\frac{1}{2}\left(\mathbb{T}^{(1)}\right)^{2}\right)\left(\beta_{0}+\frac{1}{2} \mathbf{H}_{\mathrm{inv}}^{(1)}\right),
\end{aligned}
$$

where $\mathbf{H}_{\text {inv }}^{(\ell)}$ are (canonically) $S L(2)$-invariant operators with kernels that are functions of the conformal ratio $\tau$. The operators $\mathbb{T}^{(i)}$ commute with $S_{-}^{(0)}$ and $S_{0}^{(0)}$ and are defined by the following equations:

$$
\begin{aligned}
& {\left[S_{+}^{(0)}, \mathbb{T}^{(1)}\right]=\left[\mathbf{H}_{\text {inv }}^{(1)}, z_{1}+z_{2}\right],} \\
& {\left[S_{+}^{(0)}, \mathbb{T}^{(2)}\right]=\left[\mathbf{H}_{\text {inv }}^{(2)}, z_{1}+z_{2}\right], \quad\left[S_{+}^{(0)}, \mathbb{T}_{1}^{(1)}\right]=\left[\mathbb{T}^{(1)}, z_{1}+z_{2}\right] .}
\end{aligned}
$$

Once again, these equations define the kernels up to $S L(2)$ (canonically) invariant terms. Explicit formulas for all relevant $\mathbb{T}$ - and $\mathbb{X}$-type operators can be found in Ref. [15].

Finally the result in $\overline{\mathrm{MS}}$-scheme is given by the inverse transformation

$$
\mathbb{H}(a)=\mathbb{U} \mathbf{H}(a) \mathbb{U}^{-1} .
$$




\subsection{Invariant kernel}

To complete our result for the evolution kernel in equations (3.23) we need to find expressions for the canonically invariant kernels $\mathbf{H}_{\text {inv }}^{(1,2,3)}$. For the given solution of the non-invariant part the invariant part is defined such that its spectrum reads

$$
\mathbf{H}_{\text {inv }}\left(z_{1}-z_{2}\right)^{N}=f\left(j_{N}\right)\left(z_{1}-z_{2}\right)^{N}
$$

where $j_{N}=N+2$ is the conformal spin and the eigenvalues $f\left(j_{N}\right)$ are defined by

$$
\gamma(N)=f\left(N+2+\bar{\beta}(a)+\frac{1}{2} \gamma(N)\right)=f\left(j_{N}+\bar{\beta}(a)+\frac{1}{2} \gamma(N)\right) .
$$

Recently it was shown that in the large spin limit $j_{N} \rightarrow \infty$ this function $f\left(j_{N}\right)$ is symmetric under reflection $j_{N} \rightarrow 1-j_{N}[16-18]$

$$
f\left(j_{N}\right)=2 \Gamma_{\text {cusp }} \ln j_{N}+f_{0}+\sum_{n=1}^{\infty} \frac{f_{n}\left(\ln j_{N}\right)}{\left(j_{N}\left(1-j_{N}\right)\right)^{n}} .
$$

To three-loop accuracy it can be easily obtained from the anomalous dimensions to the same accuracy [4] and is given in terms of nested harmonic sums [19] up to weight five. The leading asymptotic terms $\Gamma_{\text {cusp }}$ - the cusp anomalous dimension [16] - and $f_{0}$ are the same as for the anomalous dimensions itself [4], the two functions differ just in the sub-leading structure.

With the help of this asymptotic form we easily find the generic form for the invariant kernel

$$
\begin{aligned}
\mathbf{H}_{\text {inv }} \mathscr{O}\left(z_{1}, z_{2}\right)= & \chi_{o} \mathscr{O}\left(z_{1}, z_{2}\right)+\Gamma_{\text {cusp }} \int_{0}^{1} d \alpha \frac{\bar{\alpha}}{\alpha}\left(2 \mathscr{O}\left(z_{1}, z_{2}\right)-\mathscr{O}\left(z_{12}^{\alpha}, z_{2}\right)-\mathscr{O}\left(z_{1}, z_{21}^{\beta}\right)\right) \\
& +\int_{0}^{1} d \alpha \int_{0}^{\bar{\alpha}} d \beta\left(\chi_{\text {inv }}^{+}(\tau)+\chi_{\text {inv }}^{-}(\tau) \mathbb{P}_{12}\right) \mathscr{O}\left(z_{12}^{\alpha}, z_{21}^{\beta}\right),
\end{aligned}
$$

with $\chi_{0}=f_{0}+2 \Gamma_{\text {cusp }}$ and $\mathbb{P}_{12} f\left(z_{1}, z_{2}\right)=f\left(z_{2}, z_{1}\right)$. For $n_{f}=4$ we find

$$
\Gamma_{\text {cusp }}=5.33 a+42.8 a^{2}+429 a^{3}+O\left(a^{4}\right), \quad \chi_{0}=2.67 a-2.12 a^{2}-377 a^{3}+O\left(a^{4}\right) .
$$

The functions $\chi_{\text {inv }}^{ \pm}(\tau)$ are motivated by the well-known general decomposition

$$
\gamma(N)=\gamma^{+}(N)+(-1)^{N} \gamma^{-}(N), \quad f\left(j_{N}\right)=f^{+}\left(j_{N}\right)+(-1)^{j_{N}} f^{-}\left(j_{N}\right) .
$$

and are fixed by the conditions

$$
\int_{0}^{1} d \alpha \int_{0}^{\bar{\alpha}} d \beta \chi_{\text {inv }}^{ \pm}(\tau)(1-\alpha-\beta)^{N}=f_{\text {sub }}^{ \pm}\left(j_{N}\right)
$$

where $f_{\text {sub }}^{ \pm}\left(j_{N}\right)$ denotes the $f^{ \pm}$- functions minus subtraction of the leading terms $\sim \Gamma_{\text {cusp }}, f_{0}$. Eq. (3.32) can be solved for the kernel function via

$$
\chi_{\text {inv }}^{ \pm}(\tau)=\frac{1}{2 \pi i} \int_{c-i \infty}^{c+i \infty} d N(2 N+3) f_{\text {sub }}^{ \pm}\left(j_{N}\right) P_{N+1}\left(\frac{1+\tau}{1-\tau}\right),
$$

where $P_{N}(x)$ are Legendre polynomials and $c$ has to be chosen such that all poles of the integrand lie to the left of the contour. 

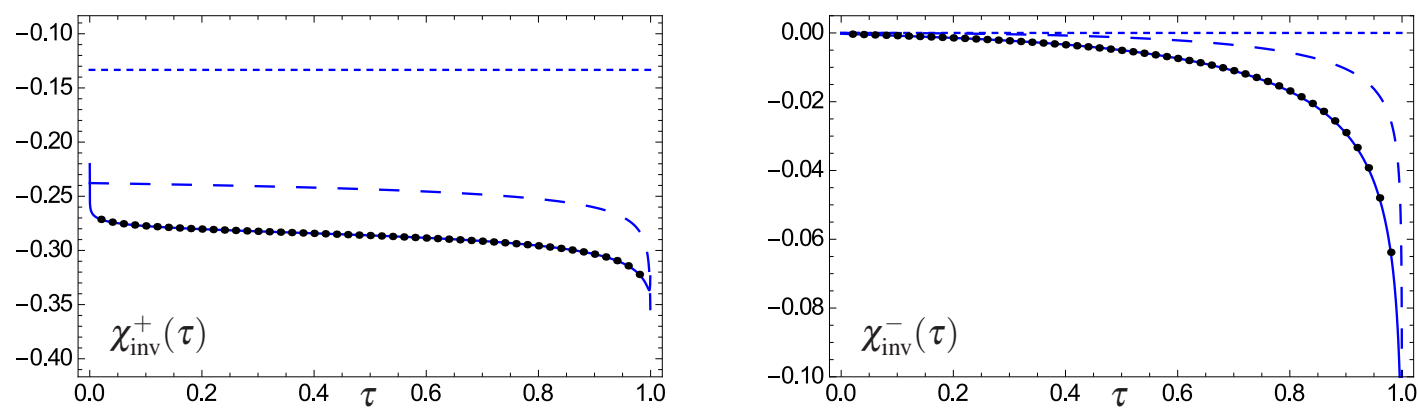

Figure 1: The LO result (short dashes) is compared to the NLO (long dashes) and NNLO (solid curves), for $\alpha_{s} / \pi=0.1$ Our results are in perfect agreement with the NNLO results using exact $\mathscr{O}\left(a^{3}\right)$ functions obtained by the numerical integration of Eq. (3.33), shown by black dots.

To LO and NLO accuracy the inversion (3.33) is simple enough to allow for a purely analytic solution. At three-loop accuracy however, we need to employ a semi-analytic solution, with a partial result given as a two-parameter fit to the data obtained from a numerical integration of equation (3.33). Our simple fit turns out to be within $98 \%$ accuracy compared to the "exact" numerical solution of equation (3.33). Our findings for the functions $\chi_{\text {inv }}^{ \pm}(\tau)$ for $n_{f}=4$ and $a=$ 1/40 are visualized in Fig. 1.

For explicit all- $n_{f}$ results we again need to refer the reader to Ref. [15].

\section{Results for local operators}

To present explicit results for the anomalous dimension matrix $\gamma_{m k}^{m^{\prime} k^{\prime}}$ we first need to choose a basis of operators. Most convenient for our application is the choice

$$
O_{n k}=(\overleftarrow{\partial}+\vec{\partial})^{k} \bar{q}(x) C_{n}^{3 / 2}\left(\frac{\overleftarrow{D}-\vec{D}}{\overleftarrow{\partial}+\vec{\partial}}\right) q(x)
$$

where $C_{n}^{3 / 2}(x)$ are Gegenbauer polynomials and the corresponding set of dual polynomials reads

$$
\tilde{P}_{n k}\left(z_{1}, z_{2}\right)=\tilde{\omega}_{n k}\left(S_{+}^{(0)}\right)^{k-n}\left(z_{1}-z_{2}\right)^{n}, \quad \tilde{\omega}_{n k}=2 \frac{2 n+3}{(k-n) !} \frac{\Gamma(n+2)}{\Gamma(n+k+4)},
$$

For this set of operators the second index $k^{2}$ labels the Poincare representation as it counts the number of total derivatives and therefore there is no mixing w.r.t. to this index

$$
\gamma_{n k}^{n^{\prime} k^{\prime}} \equiv \gamma_{n n^{\prime}}
$$

\footnotetext{
${ }^{2}$ Note that $k>n$
} 
Another major advantage of this choice is that the anomalous dimension matrix takes lower triangular form

$$
\gamma_{n n^{\prime}}=0, \text { if } n<n^{\prime},
$$

and hence the diagonal elements are given by the eigenvalues, alias the known forward anomalous dimensions [4]. The desired off-diagonal elements are given by the matrix elements of the SL(2)non-invariant evolution kernel, which we have determined exactly up to three-loop accuracy. Thus the anomalous dimension matrix reads $\gamma_{n n^{\prime}}=\delta_{n n^{\prime}} \gamma_{n}^{\mathrm{D}}+\gamma_{n n^{\prime}}^{\mathrm{ND}}$ with

$$
\gamma_{n n^{\prime}}^{\mathrm{ND}}=\left.\left(\partial_{1}+\partial_{2}\right)^{k} C_{n}^{3 / 2}\left(\frac{\partial_{1}-\partial_{2}}{\partial_{1}+\partial_{2}}\right) \mathbb{H}_{n i n v} \tilde{P}_{n^{\prime} k^{\prime}}\left(z_{1}, z_{2}\right)\right|_{z_{1}=z_{2}=0}, \quad n>n^{\prime}
$$

For the first few elements of the mixing matrix (for $n_{f}=4$ ) we obtain up to the order $O\left(a^{3}\right)$

$$
\gamma_{n n^{\prime}}=a\left(\begin{array}{ccccc}
0 & 0 & 0 & 0 & 0 \\
0 & 7.1 & 0 & 0 & 0 \\
0 & 0 & 11 & 0 & 0 \\
0 & 0 & 0 & 14 & 0 \\
0 & 0 & 0 & 0 & 16
\end{array}\right)+a^{2}\left(\begin{array}{ccccc}
0 & 0 & 0 & 0 & 0 \\
0 & 71 & 0 & 0 & 0 \\
11 & 0 & 100 & 0 & 0 \\
0 & 23 & 0 & 120 & 0 \\
-3.5 & 0 & 25 & 0 & 130
\end{array}\right)+a^{3}\left(\begin{array}{ccccc}
0 & 0 & 0 & 0 & 0 \\
0 & 610 & 0 & 0 & 0 \\
180 & 0 & 840 & 0 & 0 \\
0 & 290 & 0 & 1000 & 0 \\
-13 & 0 & 300 & 0 & 1100
\end{array}\right)
$$

We conclude with the remark that non-zero off-diagonal elements appear for the first time at twoloop accuracy. Moreover our findings are in agreement with the known $O\left(a^{2}\right)$ off-diagonal anomalous dimension $[6,8]$.

\section{Acknowledgments}

The author would like to thank the organizers of the QCD evolution 2017 workshop for hospitality and financial support. The work was supported by Deutsche Forschungsgemeinschaft (DFG) with the grant SFB/TRR 55 .

\section{References}

[1] J. Dudek et al., Physics Opportunities with the 12 GeV Upgrade at Jefferson Lab, Eur. Phys. J. A48 (2012) 187, [1208.1244].

[2] D. Boer et al., Gluons and the quark sea at high energies: Distributions, polarization, tomography, 1108.1713.

[3] A. Accardi et al., A Critical Appraisal and Evaluation of Modern PDFs, Eur. Phys. J. C76 (2016) 471, [1603.08906].

[4] S. Moch, J. A. M. Vermaseren and A. Vogt, The Three loop splitting functions in QCD: The Nonsinglet case, Nucl. Phys. B688 (2004) 101-134, [hep-ph / 0403192$].$

[5] S. Moch, B. Ruijl, T. Ueda, J. A. M. Vermaseren and A. Vogt, Four-Loop Non-Singlet Splitting Functions in the Planar Limit and Beyond, 1707.08315. 
[6] D. Mueller, Conformal constraints and the evolution of the nonsinglet meson distribution amplitude, Phys. Rev. D49 (1994) 2525-2535.

[7] D. Mueller, Restricted conformal invariance in $Q C D$ and its predictive power for virtual two photon processes, Phys. Rev. D58 (1998) 054005, [hep-ph/970 4406 ].

[8] A. V. Belitsky and D. Mueller, Predictions from conformal algebra for the deeply virtual Compton scattering, Phys. Lett. B417 (1998) 129-140, [hep-ph/ 9709379 ].

[9] D. Mueller, Constraints for anomalous dimensions of local light cone operators in phi**3 in six-dimensions theory, Z. Phys. C49 (1991) 293-300.

[10] V. M. Braun and A. N. Manashov, Evolution equations beyond one loop from conformal symmetry, Eur. Phys. J. C73 (2013) 2544, [1306.5644].

[11] V. M. Braun, A. N. Manashov, S. Moch and M. Strohmaier, Two-loop conformal generators for leading-twist operators in QCD, JHEP 03 (2016) 142, [1 601 . 05937].

[12] V. M. Braun and A. N. Manashov, QCD evolution equations from conformal symmetry, Int. J. Mod. Phys. Conf. Ser. 37 (2015) 1560048, [1408.6648].

[13] T. Banks and A. Zaks, On the Phase Structure of Vector-Like Gauge Theories with Massless Fermions, Nucl. Phys. B196 (1982) 189-204.

[14] A. Hasenfratz and P. Hasenfratz, The Equivalence of the SU(N) Yang-Mills theory with a purely fermionic model, Phys. Lett. B297 (1992) 166-170, [hep-lat/9207017].

[15] V. M. Braun, A. N. Manashov, S. Moch and M. Strohmaier, Three-loop evolution equation for flavor-nonsinglet operators in off-forward kinematics, JHEP 06 (2017) 037, [1703. 09532].

[16] B. Basso and G. P. Korchemsky, Anomalous dimensions of high-spin operators beyond the leading order, Nucl. Phys. B775 (2007) 1-30, [hep-th / 0612247$].$

[17] L. F. Alday, A. Bissi and T. Lukowski, Large spin systematics in CFT, JHEP 11 (2015) 101, [1502.07707].

[18] L. F. Alday and A. Zhiboedov, An Algebraic Approach to the Analytic Bootstrap, JHEP 04 (2017) $157,[1510.08091]$.

[19] J. A. M. Vermaseren, Harmonic sums, Mellin transforms and integrals, Int. J. Mod. Phys. A14 (1999) 2037-2076, [hep-ph/9806280]. 